\title{
One century of cosmic rays - A particle physicist's view
}

\author{
Christine Sutton ${ }^{\mathrm{a}}$ \\ CERN, 1211 Geneva 23, Switzerland
}

\begin{abstract}
Experiments on cosmic rays and the elementary particles share a common history that dates back to the $19^{\text {th }}$ century. Following the discovery of radioactivity in the 1890 s, the paths of the two fields intertwined, especially during the decades after the discovery of cosmic rays. Experiments demonstrated that the primary cosmic rays are positively charged particles, while other studies of cosmic rays revealed various new sub-atomic particles, including the first antiparticle. Techniques developed in common led to the birth of neutrino astronomy in 1987 and the first observation of a cosmic $\gamma$-ray source by a ground-based cosmic-ray telescope in 1989.
\end{abstract}

\section{Introduction}

"There are more things in heaven and earth than are dreamt of in your philosophy..." (Hamlet, William Shakespeare).

The modern fields of cosmic-ray studies and experimental particle physics have much in common, as they both investigate the high-energy interactions of subatomic particles. Today, cosmic-ray experiments are finding evidence for primary cosmic rays with energies as high as $10^{20} \mathrm{eV}$ and, while terrestrial accelerators cannot reach such high energies, the Large Hadron Collider at CERN is approaching interesting cosmic-ray energies, with collisions at $13 \mathrm{TeV}\left(1.3 \times 10^{13} \mathrm{eV}\right)$ in the centre of mass in sight this year. The two fields have an intertwined history that goes back beyond the discovery of cosmic rays more than a century ago. This account takes a look at a few highlights of that common history, from the perspective of an experimental particle physicist, with elements in the history of cosmic-ray physics interspersed with brief "interludes" on contemporary developments in the physics of elementary particles.

\section{Some pre-history: Common roots in the $19^{\text {th }}$ century}

Both fields have a shared ancestry that goes back to Charles-Augustin de Coulomb who reported in 1785 on his discovery of the spontaneous discharge of isolated electrified bodies. Over the following decades, electricity came under the scrutiny of many scientists, such as Michael Faraday, who made a number of notable contributions. He introduced the word "ion" in 1834 for the entities that he found to travel between electrodes in his experiments, and also investigated the passage of electricity through rarefied air in glass tubes. This was to become an important subject of study in several countries, and the development of improved vacuum pumps to

\footnotetext{
a e-mail: christine.sutton@cern.ch
}

remove the air led to the discovery of "cathode rays" emitted from the cathode at one end of the tube. In 1879, in the course of investigations in England, William Crookes discovered that the speed of the discharge along the length of a vacuum tube decreased as he reduced the pressure. This indicated that ionization of the air was causing the discharge.

The last few years of the 19th century saw a burst of discoveries that not only cast light on the effect that Crookes had observed, but also opened up new worlds for investigation. In Germany, in 1895, Wilhem Röntgen discovered X-rays while investigating cathode rays. A year later in France, Henri Becquerel discovered radioactivity while investigating the production of $\mathrm{X}$ rays. Then in 1897 , J J Thomson in England, among others, pinned down the nature of cathode rays, finding that they consist of particles much smaller than the atom. This marked the discovery of the electron - the first known elementary particle. Radioactivity had in the meantime become the subject of some intense investigation and by 1899 Ernest Rutherford had found two associated forms of radiation, which he called $\alpha$ and $\beta$. A year later, Philippe Villard discovered a third, penetrating radiation, which Rutherford named $\gamma$-rays.

This period of discovery also saw developments in experimental techniques that were to have an important influence in this story. In 1895, Charles Wilson invented the cloud chamber with a view to studying some of the intriguing atmospheric effects he had observed in the Scottish mountains. He also worked on improving the insulation and hence the sensitivity of electroscopes, as did Julius Elster and Hans Geitel, and in 1900 he even suggested that ionization of air could be caused by penetrating extra-terrestrial radiation.

\section{Discovery: Adventures in the early $20^{\text {th }}$ century}

The first ten years or so of the $20^{\text {th }}$ century saw a number of efforts to take electroscopes away from the ionizing 


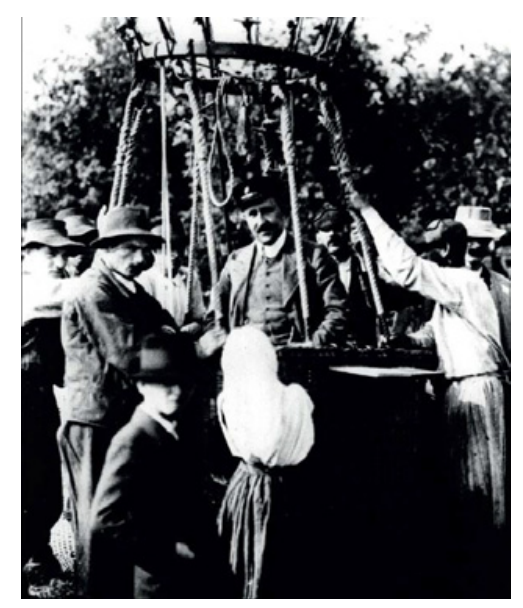

Figure 1. Hess in the basket of his balloon, sometime in 1912.

effects of the Earth's natural radioactivity. In 1900-1903 Franz Linke made 12 balloon flights during his PhD studies at Berlin University. Measuring the ionization he found "between 1 and $3 \mathrm{~km}$ the same amount; above it is larger... up to a factor of 4 (at $5500 \mathrm{~m})$, The uncertainties in the observations [...] only allow the conclusion that the reason for the ionization has to be found first in the Earth". The measurement was good, but Linke drew the wrong conclusions. In 1909 Theodor Wulf perfected the portable electroscope and found that the amount of radiation at the top of the Eiffel Tower $(300 \mathrm{~m})$ is "not even half of its ground value". The same year, Karl Bergwitz observed during a balloon flight that the ionization at $1300 \mathrm{~m}$ is about $24 \%$ of the value on the ground. Alfred Gockel flew up to $3000 \mathrm{~m}$, finding similar results. He also introduced the term "kosmische Strahlung", or "cosmic radiation". Taking a different approach, in 1911-1912 Domenico Pacini found that the rate of ionization at $3 \mathrm{~m}$ below water is $20 \%$ lower than at the surface: "[It] appears...that a sizable cause of ionization exists in the atmosphere originating from penetrating radiation".

Around the same time, Victor Hess (Fig. 1) took measurements on two flights in 1911 and found "no essential change" in the amount of radiation compared with near the ground. This implied the existence of a radiation source in addition to $\gamma$-rays from radioactive decays in the Earth's crust. Then, on 7 August 1912, on the last of a series of balloon flights using two Wulf electroscopes, he observed: "In both $\gamma$-ray detectors the values at the greatest altitude are about 22-24 ions higher than at the ground.... By 3000 to $4000 \mathrm{~m}$ the increase amounts to 4 ions, and at 4000 to $5200 \mathrm{~m}$ fully to 16 to 18 ions, in both detectors.... The results of the present observations seem to be most readily explained by the assumption that a radiation of very high penetrating power enters our atmosphere from above." This observation by Hess is now widely regarded as the discovery of cosmic rays.

\subsection{First interlude}

While the desire to make measurements away from terrestrial radioactivity had led some scientists to the discovery of cosmic rays, others such as Rutherford were turning radioactivity into a tool to learn more about the structure of matter. In 1911 he published his analysis of the wide-angle scattering of $\alpha$-particles from gold leaf measured by his colleagues Hans Geiger and Ernest Marsden. He concluded that the results demonstrated that the positive charge of the atom was concentrated in a tiny, central region - the nucleus. This was also the year that Wilson made the first observation of the tracks of charged particles in a cloud chamber.

From 1914-18, the First World War saw many physicists required to turn from curiosity-driven research to apply their skills for the war effort - or indeed to fight for their countries. Rutherford managed to continue his pre-war studies to a limited extent and after the war he was able, in 1919, to announce the discovery of the proton as a primary constituent of the nucleus. The age of classical physics was well and truly crumbling, and the golden era of quantum mechanics was about to begin. Among these developments, in a theory of the electron that brought together quantum mechanics and Einstein's special relativity, in 1927 Paul Dirac predicted the existence of the "anti-electron". And in attempting to understand energy conservation in radioactivity, in 1930 Wolfgang Pauli declared: "I have done a terrible thing. I have postulated a particle that cannot be detected". He had hypothesized the particle that was later named the neutrino.

\section{What are they? Discoveries up to around 1930}

So just what was the extra-terrestrial radiation that Hess and others had discovered? By the 1920s, Robert Millikan in the US was convinced that the primary cosmic rays were "ultra" $\gamma$-rays released in the synthesis of heavier elements from hydrogen in space - he called them "the birth-cry of atoms" and promoted the name "cosmic rays". However, during 1926-7 Jacob Clay measured the ionization from cosmic radiation at varying latitudes. Travelling by sea between Amsterdam and the Dutch East Indies, he found a fall-off in the flux of about $15 \%$. This suggested that the primary cosmic rays are influenced by the Earth's magnetic field and must be charged particles, rather than $\gamma$-rays.

An important breakthrough in techniques came in 1929 when Walter Bothe and Werner Kohlhörster used the coincidence technique with Geiger-Müller (G-M) tubes to detect single charged particles in cosmic radiation. The G-M tubes were connected to fibre electrometers, which were photographed to show coincidences. The technique was to inspire Bruno Rossi, among others, and in 1930 he invented a coincidence circuit using vacuum triode valves. This was the first practical AND circuit. Rossi used it to confirm the results that most of the cosmic rays being detected were charged particles and had too much energy to be created from $\gamma$-rays emitted in the synthesis of elements, as Millikan proposed. A year later, he presented his results in the introductory talk at a conference in Rome, which was attended by Millikan. Rossi late recalled: "Millikan clearly resented having his beloved theory torn to pieces by a mere youth, so much so that from that moment on he refused to recognise my existence." 
Table 1. Particles of matter and their antiparticles, 1934 (hypothesized particles are indicated in italic font).

\begin{tabular}{|l|l|l|}
\hline Particles & $\begin{array}{l}\text { proton } \\
\text { neutron }\end{array}$ & $\begin{array}{l}\text { electron } \\
\text { neutrino }\end{array}$ \\
\hline Antiparticles & $\begin{array}{l}\text { antiproton } \\
\text { antineutron }\end{array}$ & $\begin{array}{l}\text { positron } \\
\text { antineutrino }\end{array}$ \\
\hline
\end{tabular}

Around the same time, Arthur Compton began to lead a worldwide survey of geographic variations. These comprehensively confirmed the latitude effect that Clay had discovered and, hence, that cosmic rays are charged particles. Rossi meanwhile had predicted an asymmetry related to the sign of the charge of cosmic rays - the east-west effect - which would be greatest near the geomagnetic equator. In 1933, in work in Eritrea, Rossi confirmed the effect, as did other measurements by Tom Johnson, and by Luis Alvarez and Compton in Mexico. It was now clear that the primary cosmic rays are positively charged particles.

In the meantime, as different detection techniques evolved, so did the study of the secondary cosmic rays reaching the Earth's surface. In 1927 Dmitry Skobeltzyn in Leningrad had placed a cloud chamber in a strong magnetic field $(1.5 \mathrm{kG})$ and observed some tracks that were almost straight, implying that they were made by energetic particles - probably cosmic rays. In the US, Millikan suggested to Carl Anderson in 1930 that he should study the energy of cosmic-ray particles in a cloud chamber. Two years later, Anderson found evidence for positively charged lightly-ionizing particles, just like "positive electrons". This marked the discovery of the "anti-electron" - or positron - that Dirac had predicted in 1927.

\subsection{Second interlude}

The positron was not the only fundamental particle to be discovered in 1932. In Rutherford's group in Cambridge, James Chadwick found evidence for a neutral constituent in the atomic nucleus. This was the neutron, which together with the proton accounts for all of the mass of the nucleus and most of the mass of the atom. Following this discovery, and working on an analogy to Dirac's theory of radiation by electrons, in 1934 Enrico Fermi wrote down a theory of $\beta$ decay in which he also included Pauli's hypothesized particle - which he named the neutrino. By this point it seemed that a description of matter at a fundamental level might require only four elementary particles - and their antiparticles - as shown in Table 1.

The same year, in the Soviet Union another discovery was made that would later become the basis of an important experimental technique in both cosmic-ray and particle physics. Pavel Cherenkov, working under Sergei Vavilov, observed a new phenomenon - the radiation of light by charged particles travelling faster than light through a medium. This became known as "Cherenkov" radiation but, rather like Wilson's invention of the cloud chamber, it would be some time before Cherenkov detectors began to play a significant role in experiments.

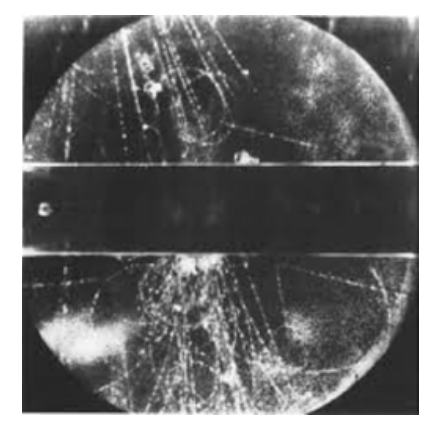

Figure 2. One of the "V" particles discovered by Rochester and Butler in 1947 [1]. The inverted V, visible to the right just below the plate across the chamber, is formed by the tracks of the decay products of a neutral $\mathrm{K}$.

\section{What are they? Discoveries up to around 1950}

The discovery of the positron in cosmic rays proved to be a turning point in studies of the nature of matter, with several new particles emerging in cosmic-ray experiments over the next two decades. In 1937, again using a cloud chamber, Anderson and Seth Neddermeyer found another new particle, this time with a mass between that of the proton and electron. It was dubbed the mesotron, but is now known as the muon - a heavier version of the electron, it forms the penetrating component of secondary cosmic rays. A decade later, using nuclear emulsions at the Pic du Midi, Cecil Powell and colleagues discovered a different, more strongly interacting particle in 1947. They also found that this particle, which became known as the pion, decayed to the muon and eventually to an electron: $\pi \rightarrow \mu \rightarrow$ e. Meanwhile, in cloud-chamber studies in Manchester, George Rochester and Clifford Butler discovered the "V" particles; with about half the proton's mass, they decayed to leave a distinctive " $\mathrm{V}$ " in the detector (Fig. 2). In 1951, Butler and colleagues found a neutral V slightly heavier than the proton. Later, these Vs became known as the "strange" particles, $\mathrm{K}$ and $\Lambda$.

\subsection{Third interlude}

To follow up these discoveries, in the 1950-60s, particle accelerators began to provide "man-made" cosmic rays, revealing many more short-lived particles. CERN, the European Organization for Nuclear Research was born in 1954 to bring together European scientists in the aftermath of the Second World War. In 1959, the Proton Synchrotron - which today is still a main cog in CERN's accelerator complex - started up, and for a time provided the highest machine-made energies in the world, at $25 \mathrm{GeV}$. The collisions of accelerated protons produced cosmic-ray-like interactions under controlled conditions - albeit at a lower energy - and provided a subatomic laboratory to study the particles found in cosmic radiation. In a golden decade discoveries came thick and fast, including:

- 1955 Owen Chamberlain and colleagues discover the antiproton at the Bevatron, at what is now Berkeley Lab.

- 1956 Fred Reines and Clyde Cowan detect neutrinos for the first time, emitted from a nuclear reactor. 
Table 2. Particles of matter 1964.

\begin{tabular}{|c|c|}
\hline $\begin{array}{c}\text { up quark } \\
\text { down quark }\end{array}$ & $\begin{array}{c}\text { electron } \\
\text { electron neutrino }\end{array}$ \\
\hline strange quark & $\begin{array}{c}\text { muon } \\
\text { muon neutrino }\end{array}$ \\
\hline
\end{tabular}

- 1962 Leon Lederman, Melvin Schwartz and Jack Steinberger at the Brookhaven Laboratory discover that there are two types of neutrino - one associated with the electron, the other with the muon.

- 1964 Murray Gell-Mann and George Zweig independently propose a substructure for protons and many other particles, which Gell-Mann calls "quarks".

With the work of Gell-Mann and Zweig, by 1964 the bewildering variety of particles discovered first in cosmic rays and later at particle accelerators could be described in terms of relatively few quarks and leptons, and their antiparticles - the leptons being the family containing the electron, muon and neutrinos. Written down as in Table 2, there are hints of a fourth quark, but confirmation of such a picture had to await another decade.

\section{The birth of neutrino astronomy}

Cowan and Reines had demonstrated that, contrary to Pauli's fear, neutrinos can be detected, provided there is a large enough flux and a big enough detector. In 1960, Moisey Markov suggested using Cherenkov light in a deep lake or ocean to detect atmospheric neutrinos - that is, to detect the charged muons produced by the interactions in the water of neutrinos created in cosmic air-showers. At the same time, Kenneth Greisen proposed using a water Cherenkov detector in a deep mine for neutrino astronomy. It was also around this time that Raymond Davis began the construction of his first experiment to detect neutrinos from the sun. In a seminal paper in 1964, he and John Bahcall argued the case for detecting solar neutrinos through capture by chlorine nuclei in around 400,000 litres of perchloroethylene (dry-cleaning fluid). In 1968 Davis published results from his experiment in the Homestake gold mine, which showed for the first time that the detected number of solar neutrinos was less than half the expectation from solar models. This was the beginning of the famous "solar neutrino problem" that was to lead some 30 years later to the discovery of neutrino oscillations and their explanation in terms of a small neutrino mass.

Meanwhile in 1965, two groups had detected cosmicray neutrinos: Reines using liquid scintillator in the East Rand gold mine in South Africa, and a Bombay-OsakaDurham collaboration operating in the Kolar Gold Field in India using an iron calorimeter. However it was to be two more decades before Greisen's dream came true. In 1987 two water-Cherenkov experiments detected neutrinos from the supernova SN1987a in the Large Magellanic Cloud: the Irvine-Michigan-Brookhaven (IMB) detector in the US and the Kamiokande detector in Japan. The Baksan scintillator telescope in the Caucasus also detected the supernova (Fig. 3). Neutrino astronomy was born.

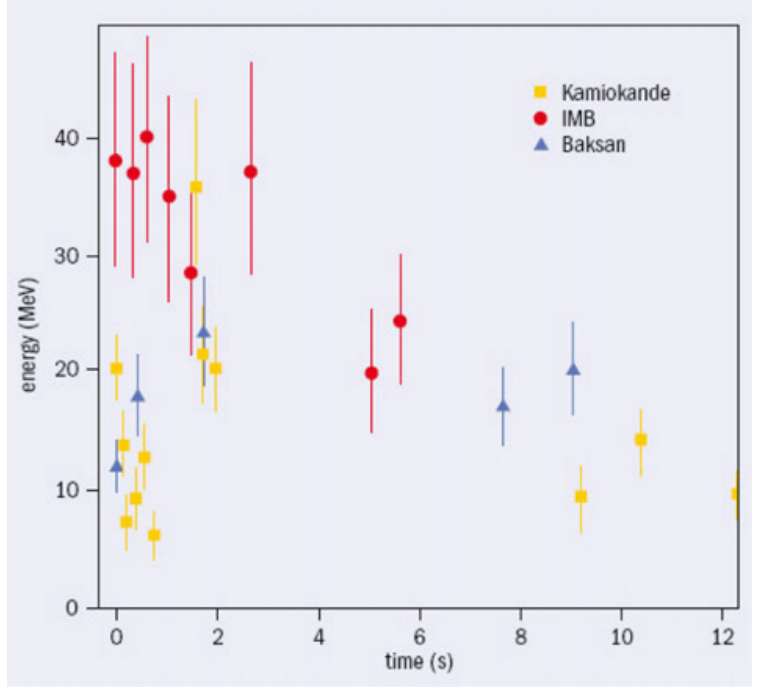

Figure 3. SN1987A neutrino events observed by Kamiokande, $\mathrm{IMB}$ and Baksan showed that the neutrino burst lasted about $13 \mathrm{~s}$ [2].

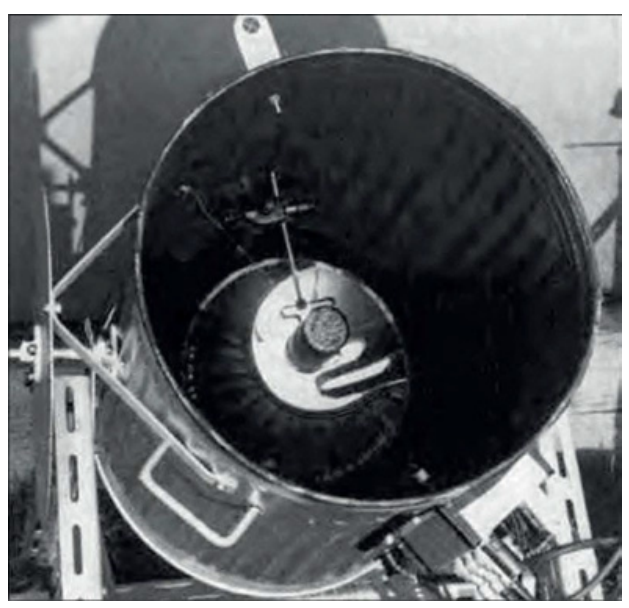

Figure 4. The detector used by Galbraith and Jelley for the first observations of atmospheric-Cherenkov radiation: a dustbin with a small parabolic mirror and phototube [3].

\section{The birth of $\gamma$-ray astronomy with cosmic rays}

The Cherenkov effect also played a key role in opening up another new window on the cosmos. In 1947, based on the general flux of cosmic radiation, Patrick Blackett in the UK estimated the contribution of Cherenkov light produced by cosmic rays passing through the atmosphere to be $0.01 \%$ of the total intensity of the night sky. A few years later, in 1953, Bill Galbraith and John Jelley pointed a dustbin containing a $60-\mathrm{cm}$ diameter mirror with a photomultiplier tube at its focus towards the sky (Fig. 4). Recording coincidences with a nearby GeigerMüller array, they succeeded for the first time in detecting Cherenkov radiation produced in the air by charged particles in extended air showers. They subsequently confirmed that they were indeed detecting Cherenkov light in measurements at the Pic du Midi. 
Table 3. Particles of matter, 1989, with hypothesized particles indicated in italic font.

\begin{tabular}{|c|c|}
\hline $\begin{array}{c}\text { up quark down } \\
\text { quark }\end{array}$ & $\begin{array}{c}\text { electron } \\
\text { electron neutrino }\end{array}$ \\
\hline $\begin{array}{c}\text { strange quark } \\
\text { charm quark }\end{array}$ & $\begin{array}{c}\text { muon } \\
\text { muon neutrino }\end{array}$ \\
\hline $\begin{array}{c}\text { top quark } \\
\text { bottom quark }\end{array}$ & $\begin{array}{c}\text { tau } \\
\text { tau neutrino }\end{array}$ \\
\hline
\end{tabular}

At the end of the decade, in 1959, Giuseppe Cocconi suggested that cosmic $\gamma$-ray sources could be detected through measurements of cosmic-ray air showers and proposed that the Crab nebula should be a strong source of high-energy $\gamma$ rays. This inspired Aleksandr Chudakov to build the first air-Cherenkov telescope in the 1960s, with 12 searchlight mirrors, each $1.5 \mathrm{~m}$ diameter, mounted on railway cars in the Crimea.

However, it was to be another two decades before Cocconi's prediction was fulfilled, when Trevor Weekes and colleagues observed $\mathrm{TeV} \gamma$ rays from the Crab nebula with the Whipple Observatory - an imaging airCherenkov telescope with a $10 \mathrm{~m}$ segmented mirror. Using data collected between December 1986 and February 1988, Weekes and his colleagues found [4]: "The observed flux $\left[1.8 \times 10^{-11}\right.$ photons $\mathrm{cm}^{-2} \mathrm{~s}^{-1}$ above $\left.0.7 \mathrm{TeV}\right]$ is only $0.2 \%$ of the cosmic-ray background... The detection of such a weak flux from a steady source with a significance of $9 \sigma$ is a milestone in the development of ground-based $\gamma$-ray astronomy." With this observation, $\gamma$-ray astronomy with imaging air-Cherenkov telescopes was born.

\section{Finale}

In 1989, the same year that Weekes and colleagues published their paper on the observation of the Crab nebula, experiments at CERN's Large Electron-Positron collider showed that there are only three types of lightweight neutrino and hence only three generations in the families of quarks and leptons. By this time, particles containing two further types of quark (charm and bottom) had been found, together with a heavier version of the electron and the muon (the tau). This left only the top quark and the tau neutrino to be discovered in a basic pattern of six particles of each type (Table 3). These missing pieces had both been discovered by the end of 2000 , thus confirming the basic elements in the current Standard Model of particle physics.

By this time, two new windows on the universe had been opened, with the first observations of cosmic phenomena using neutrinos and $\gamma$-rays found in cosmic radiation. A century after the discovery of radioactivity, which was fundamental to the development of both cosmic-ray physics and elementary-particle physics, a new era of astronomy with neutrinos and cosmic-ray photons had begun. It continues today, holding the promise of answering the century-old question: Where do cosmic rays come from?

Many people have helped me over recent years in getting to grips with cosmic-ray and neutrino physics, in particular Alan Watson and Francis Halzen. I thank them and all of the inspirational people who figured in this extremely brief look at a century of cosmic rays - including my PhD supervisor, Bill Galbraith - and the many others who I did not manage to mention.

For material on the early studies of cosmic rays, I have been helped in particular by the work of Alessandro De Angelis [5,6] and Michael Hillas [7].

Finally, I would like to dedicate this paper to the memory of two pioneers in the field of ground-based $\gamma$-ray astronomy using the imaging air-Cherenkov technique, who sadly passed away last year: Trevor Weeks (1940-2014) and Ekart Lorenz (1938-2014).

\section{References}

[1] G.D. Rochester and C.C Butler, Nature 160, 855 (1947)

[2] M. Nakahata, CERN Courier 47 no.1 23 (2007)

[3] L. Thompson, CERN Courier 52 no.6 34 (2012)

[4] T.C. Weekes, M.F. Cawley, D.J. Fegan, K.G. Gibbs, A.M. Hillas, P.W. Kwok, D.A. Lewis, D. Macomb, N.A. Porter, P.T. Reynolds, G. Vacanti, Astrophysical Journal 342, 379 (1989)

[5] A. De Angelis, Riv. Nuovo Cim. 33, 713 (2010)

[6] P. Carlson and A. De Angelis, Eur. Phys. J. H, 309 (2011)

[7] A. M. Hillas, Cosmic Rays (Pergamon Press, 1972) 